\title{
Solid-state nanopores for sensing
}

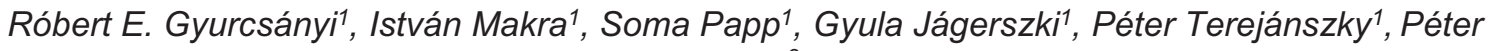 \\ Fürjes ${ }^{2}$ \\ ${ }^{1}$ MTA-BME Chemical Nanosensors Research Group, Department of Inorganic and Analytical \\ Chemistry, Budapest University of Technology and Economics, Szt. Gellért tér 4, 1111 Budapest, \\ Hungary \\ ${ }^{2}$ MTA Centre for Energy Research - Institute of Technical Physics and Materials Science, Konkoly- \\ Thege str. 29-33, $\mathrm{H}-1121$ Budapest, Hungary \\ robertgy@mail.bme.hu
}

\begin{abstract}
:
Nanopores possess an inherently built-in transduction mechanism that opens up the prospects for their use as label-free chemical sensors. Species comparable in size with the nanopore restriction that enter the nanopore are able to effectively change the physico-chemical properties of the nanopore interior, such as the conductance, which provides sensitive means for their detection. The full capability of interrogating the sample in the extremely small volume confined by the nanopore interior is achieved if a single nanopore is used for sensing, which leads to single species detection. We are going to present the application of this concept for the calibration-free sizing and counting synthetic and biological nanoparticles (viruses) with nanopipettes. Beside "passive" counting, the chemical modification of single nanopores and nanopore arrays with functional compounds and receptors offers new opportunities for selective chemical sensing with nanopores. These "unconventional" sensing concepts will be presented through the synthesis of solid-state ion channels and their application for the selective detection of ions and polyions, as well as nanoparticle displacement assays with nanopore-based sensors.
\end{abstract}

Key words: nanopores, solid-state ion channel, resistive pulse sensing, virus, ion-selective sensors

\section{Resistive pulse sensing of nanoparticles}

The most commonly used electrochemical readout in nanopore sensors is resistive pulse sensing (RPS).[1] RPS relies on monitoring changes in the applied voltage-driven ion current across a nanopore embedded in an electrically insulating membrane that separates two electrolyte chambers as the detected species are passing through the nanopore sensing zone. This implies that the detected species cause a measurable change in the pore conductance, i.e., they act as a stimulus, opening or closing the nanopore to the flux of small ions of the electrolyte solution. Since the concentration of these ions exceeds that of the target species by many orders of magnitude an inherent amplification is achieved. Clearly, the frequency of the resistive pulses and their amplitude scales with the concentration and size of the detected species, respectively, but, it is rather rare that absolute values are provided from RPS measurements. [2] We found that using the theoretical description of the resistive pulse signals combined with well characterized geometry nanopipettes enables even calibration-free sizing and quantifying of nanoparticles.[3] The performance of the methodology and the proper choice of the experimental parameters is shown through the resistive pulse sensing of synthetic nanoparticles and viruses. The nanopore-based RPS sensing excels in terms of size resolution that enables to resolve polidisperse nanoparticle samples. This is very useful to follow alteration in size (and concentration) of nanoparticle solutions subject to different separation processes, e.g. membrane filtering and centrifugation.[4]

It is important for analytical applications to understand also the fundamental limitation of nanopore sensors, i.e., the sensing zone is generally infinitesimal with respect of the probed sample volume. Consequently, the probability to have in extremely diluted solutions target species encountering the nanopore sensing zone is low, the limiting scenario being the undirected, Brownian motion.[5] Thus, in RPS the translocation frequency decreases 
with the concentration of the target species, the lowest detectable concentration being ultimately determined by the length of the measurement. Since this is limited by practical circumstances a number of approaches were considered to increase the event frequency, such as applying hydrostatic pressure, which we found to be the best option to accurately assess the concentration of target species.

\section{Chemically modified nanopores}

While RPS methods offer a wealth of information, not even the outstanding size resolution, and further information on the charge [6, 7] and shape[8] of target species may provide sufficient selectivity to assess them in complex samples. To overcome this problem nanopores were functionalized with selective receptors [9-11] to generate selective responses. Chemical functionalization also enabled the implementation of new sensing concepts. In this respect we were particularly interested in the synthesis and analytical application of selective solid state ion-channels based on nanopores. To prepare ion-selective nanopores, in a rather close analogy with the selectivity filter of biological ion channels, the inner wall of gold nanopores of a few $\mathrm{nm}$ diameter was modified with three different functional thiol and dithiolane derivatives, i.e., most importantly with an ionophore to induce selective complexation of the targeted ion $(\mathrm{Ag}+$, $\left.\mathrm{Cu}^{2+}\right), \quad[12,13]$ as well as with a cation exchanger and a perfluorocarbon derivative to confer permselectivity and hydrophobicity to the nanopore environment, respectively. Simply monitoring the membrane potential of these functionalized nanoporous membranes results in a Nernstian response to the targeted ions. Such solid-state ion channels based ionselective electrodes were shown to closely match the performance of their relevant conventional counterparts and even to provide advantages in terms of longer life time, miniaturization to the nanoscale, and selectivity. Furthermore, the potentiometric transduction mechanism could be also extended for the selective detection of nucleic acids with peptide nucleic acid functionalized nanopores.[14]

\section{Acknowledgment}

The support of the "Lendület" program of the Hungarian Academy of Sciences (LP201363) and the Bill \& Melinda Gates Foundation through the Grand Challenge Explorations Initiative (OPP1036199) is acknowledged.

\section{References}

[1] H. Bayley, C.R. Martin, Resistive-pulse sensing From microbes to molecules, Chem. Rev., 100 2575-2594, (2000); doi: 10.1021/cr980099g
[2] G.S. Roberts, S. Yu, Q. Zeng, L.C.L. Chan, W. Anderson, A.H. Colby, M.W. Grinstaff, S. Reid, R. Vogel, Tunable pores for measuring concentrations of synthetic and biological nanoparticle dispersions, Biosens. Bioelectron., 31 17-25, (2012); doi:

10.1016/j.bios.2011.09.040

[3] P. Terejanszky, I. Makra, P. Furjes, R.E. Gyurcsanyi, Calibration-less sizing and quantitation of polymeric nanoparticles and viruses with quartz nanopipets, Anal. Chem., 86 4688-4697, (2014); doi: 10.1021/ac500184z

[4] P. Terejánszky, I. Makra, A. Lukács, R.E. Gyurcsányi, Nanopipet-Based Resistive Pulse Sensing to Follow Alterations in the Size and Concentration of Nanoparticles During Membrane Filtration, Electroanal, 27 595-601, (2015); doi: 10.1002/elan.201400651

[5] L. Höfler, R.E. Gyurcsányi, Nanosensors lost in space. A random walk study of single molecule detection with single-nanopore sensors, Anal. Chim. Acta, 722 119-126, (2012); doi:

10.1016/j.aca.2012.02.010

[6] T. Ito, L. Sun, R.M. Crooks, Simultaneous determination of the size and surface charge of individual nanoparticles using a carbon nanotubebased coulter counter, Anal. Chem., 75 2399-2406, (2003); doi: 10.1021/ac023072v

[7] R. Vogel, W. Anderson, J. Eldridge, B. Glossop, G. Willmott, A Variable Pressure Method for Characterizing Nanoparticle Surface Charge Using Pore Sensors, Anal. Chem., 84 3125-3131, (2012); 10.1021/ac2030915

[8] Y. Qiu, P. Hinkle, C. Yang, H.E. Bakker, M. Schiel, H. Wang, D. Melnikov, M. Gracheva, M.E. Toimil-Molares, A. Imhof, Z.S. Siwy, Pores with Longitudinal Irregularities Distinguish Objects by Shape, ACS Nano, 9 4390-4397, (2015); doi: 10.1021/acsnano.5b00877

[9] S. Ding, C. Gao, L.-Q. Gu, Capturing Single Molecules of Immunoglobulin and Ricin with an Aptamer-Encoded Glass Nanopore, Anal. Chem., 81 6649-6655, (2009); 10.1021/ac9006705

[10] G. Jágerszki, R.E. Gyurcsányi, L. Höfler, E. Pretsch, Hybridization-modulated ion fluxes through peptide-nucleic-acid-functionalized gold nanotubes. A new approach to quantitative label-free DNA analysis, Nano Lett., 7 1609-1612, (2007); doi: 10.1021/nl0705438

[11] P. Kohli, C.C. Harrell, Z. Cao, R. Gasparac, W. Tan, C.R. Martin, DNA-Functionalized Nanotube Membranes with Single-Base Mismatch Selectivity, Science, 305 984-986, (2004); doi:

10.1126/science. 1100024

[12] G. Jágerszki, A. Takacs, I. Bitter, R.E. Gyurcsányi, Solid-State Ion Channels for Potentiometric Sensing, Angew. Chem. Int. Edit., 50 1656-1659, (2011); doi: 10.1002/anie.201003849 [13] S. Papp, G. Jagerszki, R.E. Gyurcsanyi, IonSelective Electrodes Based on Hydrophilic Ionophore-Modified Nanopores, Angew. Chem. Int. Ed., 57 4752-4755, (2018); doi:

10.1002/anie.201800954

[14] I. Makra, A. Brajnovits, G. Jágerszki, P. Fürjes, R.E. Gyurcsányi, Potentiometric sensing of nucleic acids using chemically modified nanopores,

Nanoscale, 9 739-747, (2017);

10.1039/C6NR05886H 